Book review

\title{
The Palgrave Handbook of Child Mental Health: Discourse and Conversation Studies \\ Michelle O'Reilly and Jessica Nina Lester (eds) (Macmillan, 2015)
}

\author{
Stuart Ekberg \\ Queensland University of Technology, Australia \\ stuart.ekberg@qut.edu.au
}

This large volume encompasses a range of insights for those interested in the relationship between text, talk, and social interaction and child mental health. As the editors highlight in their introduction, this handbook comes at a time when the World Health Organization estimates one in five children meet contemporary diagnostic criteria for mental health conditions. Mental health conditions, diagnoses and services are therefore either already playing a role in social lives of a significant minority of children, or have the potential to do so in the future. Understanding the social dimensions of this is critical.

Contributions to the handbook include clinical perspectives and foundational research using critical, discourse or conversation analysis. Although the contributions are diverse, the sheer size of this volume means there are more than a dozen chapters focusing on social interactions with children and families that are likely to be of interest to this journal's readership. Scholars of social interaction who are new to collaborating with healthcare professionals will also find Khalid Karim's chapter on a clinician's view of conversation analysis informative. 
In the first chapter of the book, Alessandra Fasulo maps the terrain that is covered in subsequent chapters by identifying of three clusters of existing research relating to child mental health:

1 everyday interactions involving children diagnosed with mental health conditions (especially what is currently referred to as autism spectrum disorder);

2 institutional interactions focused on assessing mental health; and

3 institutional interactions focused on delivering interventions to treat mental health conditions.

Consistent with Fasulo's overview of existing research, many chapters report studies that contribute to the first and third areas of research. For example, in considering everyday interactions, Laura Sterponi, Kenton de Kirby and Jennifer Shankey provide fascinating insights into the atypical use of pronouns by children diagnosed with autism spectrum disorder (ASD). Both pronoun avoidance and pronoun reversal (e.g. using 'you', 'he' or 'she' to refer to oneself, and ' $I$ ' to refer to others) are commonly associated with ASD. However, this chapter provides a caution against treating all instances of pronoun atypicality as clinically relevant symptoms.

Through their analysis of social interactions involving one child diagnosed with ASD, Sterponi and colleagues confirm that pronoun atypicality is salient in ASD, while also finding that there are often interactional contingencies that are relevant for understanding specific instances of this phenomenon. This shows that uniformly interpreting pronoun atypicality as a deficit misunderstands the competence of children diagnosed with ASD in using the pronominal system. In addition to having implications for clinical practice, studies like this contribute to foundational research domains in conversation analysis, such as the organization of person reference practices.

Although smaller in number, the handbook also contains contributions to understanding institutional interactions that are focused on assessing child mental health. For example, Victoria Stafford and Khalid Karim consider how problems are presented during assessments conducted in a Child and Adolescent Mental Health Service (CAMHS). Stafford and Karim highlight that the problem presentation phase is one of the only opportunities during assessments where children can voice their understanding of why they have come to use the service. Through analysis of 28 CAMHS appointments, Stafford and Karim identify how children aged

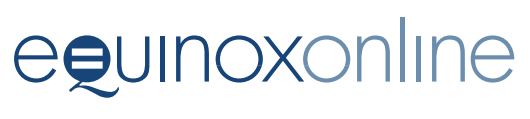


six to seventeen years old are capable of contributing to the assessment process, although many may initially claim a lack of knowledge about the reason they are attending the CAMHS. When given sufficient opportunities to do so, this study shows how children can provide valuable insights into their situation and circumstances.

The largest proportion of studies reported in the handbook examine the social interactions through which clinical interventions are delivered. For example, Jessica Lester and Michelle O'Reilly explore the use of a social thinking curriculum to engage children who have been diagnosed with ASD. This involves personifying positive behaviours as a superhero character ('Superflex') and problematic behaviours as 'unthinkable' antihero characters (e.g. 'Glassman', who has 'shattering' reactions to small problems). Having examined a large corpus of 175 hours of data, Lester and $\mathrm{O}$ 'Reilly highlight how these characterizations provide indirect ways to approach the potentially delicate task of discussing personal behaviour. Like the study by Sterponi and colleagues, this research has clinical relevance while also providing foundational insights into practices of word selection.

Beyond contributions to the three clusters of research identified by Fasulo, there are chapters in this handbook that highlight the potential for scholars of social interaction to explore a range of other areas that are relevant to child mental health. These include studies of online forums for peer support (Janet Smithson), interactions involving humanoid robots and children diagnosed with mental health conditions (Paul Dickerson and Ben Robins), and services to support children who may not have mental health conditions but are experiencing atypical circumstances that could affect their mental wellbeing (Susan Danby and colleagues).

The publication of this handbook represents considerable progress in understanding childhood mental health and social interaction across everyday and clinical settings. Notwithstanding this progress, these contributions highlight that much more research is needed to understand the social dimensions of child mental health. In particular, while research is still needed in relation to ASD, more research needs to consider areas such as:

- mental health conditions other than ASD;

- novel mental health interventions, including those that do not involve clinicians; and 
- interactional dimensions of mental wellbeing among children who do not meet diagnostic criteria for mental health conditions.

By progressing existing areas of strength and showcasing new possibilities, the bountiful research reported in this handbook charts exciting paths for future research. 